\title{
Adenylate Cyclase System Is Essential for Long-Term Facilitation at the Crayfish Neuromuscular Junction
}

\author{
D. Dixona and H. L. Atwood \\ Department of Physiology, University of Toronto, Toronto, Ontario, Canada, M5S 1A8
}

Long-term facilitation (LTF), a form of synaptic plasticity demonstrated at the crayfish neuromuscular junction, is induced by tetanic stimulation and persists for hours. LTF can be divided into 2 phases: a tetanic phase, which occurs during stimulation, and a long-lasting phase, which persists after stimulation. Activators and potentiators of CAMP (forskolin and 3-isobutyl-methyl-xanthine) produce facilitation of excitatory postsynaptic potentials, which attain approximately the amplitude of the long-lasting phase of LTF but last for a shorter time. Localized presynaptic injection of a protein inhibitor ("Walsh inhibitor") specific for the cAMPdependent protein kinase blocks the long-lasting phase of LTF at synapses near the injection site with no apparent effect on the tetanic phase. Normal LTF develops and persists at synapses of the same axon distant from the injection site. Localization of the injected inhibitor was confirmed by fluorescent tagging. Localized injection of SQ22,536, an adenylate cyclase inhibitor, also blocks the second phase of LTF near the injection site, but not at distant synapses. These experiments establish a role for adenylate cyclase activation in the long-lasting phase of LTF. The phosphatidylinositol second-messenger system is not important in LTF as inhibition of phospholipase $\mathrm{C}$ by injection of RA233, which blocks facilitatory effects of serotonin, does not affect any aspect of LTF.

Stimulus-induced changes in synaptic performance have been suggested as a possible mechanism of memory (Teyler and DiScenna, 1987). Repeated activation often leads to enhanced performance of synaptic pathways for a considerable length of time. Changes in performance lasting hours to days generally involve second-messenger systems (Schwartz and Greenberg, 1987). In some cases, protein synthesis is necessary to consolidate changes that last longer than a day (Montarolo et al., 1986; Schacher et al., 1988).

Long-term facilitation (LTF) at the crayfish neuromuscular junction is a long-lasting enhancement of synaptic transmission induced by extra impulse activity (Sherman and Atwood, 1971). It has been shown to be presynaptic in origin (Wojtowicz and

\footnotetext{
Received Jan. 11, 1989; revised May 16, 1989; accepted June 15, 1989.

This research was supported by a grant from the Medical Research Council of Canada and by an Ontario Graduate Scholarship (to D.D.). Marianne HegströmWojtowicz provided technical assistance and helped with the preparation of the manuscript. Advice from Drs. M. Wojtowicz, M. Charlton, J. MacDonald, and $P$. Pennefather, all at the University of Toronto, is gratefully acknowledged.

Correspondence should be addressed to Dr. H. L. Atwood at the above address.

a Present address: Department of Ophthalmology, University of California, San Francisco, CA 94143-0730.

Copyright (C 1989 Society for Neuroscience $0270-6474 / 89 / 124246-07 \$ 02.00 / 0$
}

Atwood, 1986) and is of physiological importance to the animal (Atwood and Wojtowicz, 1986). Once induced, enhanced transmission persists for several hours and has been shown to last for up to 1 day (Lnenicka and Atwood, 1985).

The possibility that second messengers play a role in LTF, particularly the long-lasting phase, has not been carefully examined at the crayfish neuromuscular junction. However, compounds that activate adenylate cyclase, elevate cAMP, or activate A-kinase produce a long-lasting facilitation of excitatory postsynaptic potentials (EPSPs) (Enyeart, 1981; Dixon and Atwood, 1988). It is therefore possible that cAMP-mediated reactions are involved in the long-lasting phase of LTF.

The adenylate cyclase pathway has been well established up to the point of A-kinase activation (Ross and Gilman, 1980). There are many compounds available that act on this pathway, either by enhancing or by inhibiting various reactions in it. Using such compounds, we have investigated the adenylate cyclase pathway with respect to its effect on transmitter release in crayfish and examined its role in the production of LTF. We have found that the long-lasting phase of LTF requires this pathway. We have also observed that treatments that interfere with the phosphatidylinositol system do not affect the longlasting phase of LTF, though they eliminate the late phase of serotonin facilitation (Dixon and Atwood, 1987, 1989; Dixon, 1988).

\section{Materials and Methods}

Preparation. The opener muscle in the first walking leg of the crayfish Procambarus clarkii (Girard) was used as the experimental preparation. This preparation has been described previously, as have the basic recording techniques (Wojtowicz and Atwood, 1984). Procedures for injection of materials into motor axon terminals have been described by Wojtowicz and Atwood (1985) and Dixon and Atwood (1989); additional details are given below. The preparation was continuously superfused with Van Harreveld's solution (Van Harreveld, 1936) containing $1 \mathrm{~mm}$ HEPES buffer. Temperature of the preparation chamber was continuously monitored and maintained between 12 and $12.5^{\circ} \mathrm{C}$.

In LTF experiments, EPSPs were measured before, during, and after $20-\mathrm{Hz}$ stimulation. Before and after $20-\mathrm{Hz}$ stimulation, EPSP samples were taken every $5 \mathrm{~min}$; each sample consisted of 200 events at $5 \mathrm{~Hz}$, of which the last 128 were averaged. An extra sample was taken at 2.5 min after the tetanus. During the $20-\mathrm{Hz}$ tetanus, samples were taken every $2.5 \mathrm{~min}$.

Compounds that enhance cAMP. 3-Isobutyl-methyl-xanthine (IBMX) and forskolin were mixed into crayfish solution prior to the experiment at concentrations of $100 \mu \mathrm{M}$ and $1 \mu \mathrm{M}$, respectively. After baseline recordings had been obtained from pre and postsynaptic sites, these compounds were applied by superfusion.

Inhibitory compounds. Phosphokinase A ("Walsh") inhibitor (PKI), SQ22,536 (adenylate cyclase inhibitor), and RA233 (phospholipase C inhibitor) were mixed into an electrode filling solution containing 0.05 M TAPS (tris hydroxy-methyl methylamino-propane sulfonic acid) buffer and $0.95 \mathrm{M}$ potassium acetate. The final concentration for SQ22,536 


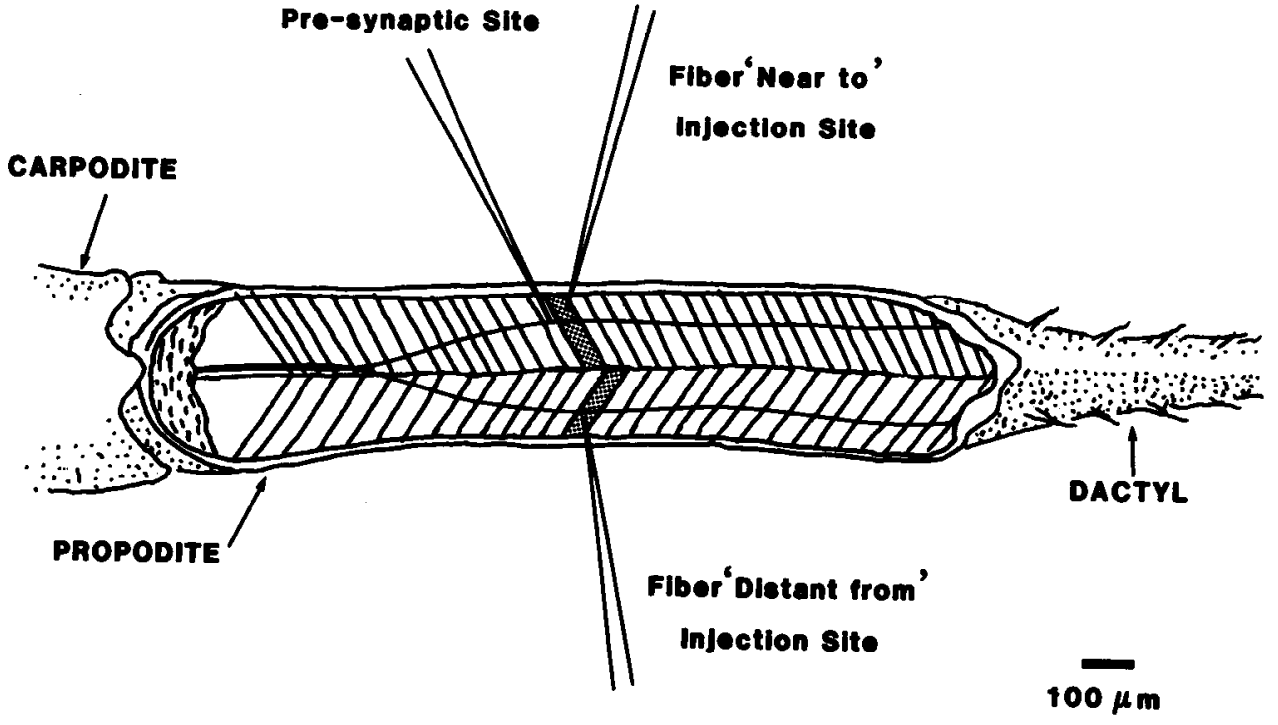

Figure 1. Method of recording changes in synaptic transmission at different postsynaptic locations with respect to presynaptic injection of active matcrials. Schematic representation of the crayfish opener muscle preparation shows the location of the presynaptic recording and injection site. Two postsynaptic sites (filled fibers) are separated by $2 \mathrm{~mm}$ of axon length. Since spread of injected materials is slow and limited, the recordings at the distant site serve as controls for responses at the proximate site. and RA233 was $1 \mathrm{~mm}$, for PKI $5 \mathrm{mg} / \mathrm{ml}$. The $\mathrm{pH}$ of the solution was set at 7.4. Solutions containing the inhibitors were used to tip-fill $10-$ $12 \mathrm{M} \Omega$ microelectrodes for presynaptic penetration. Once the presynaptic electrode was in place, 2 postsynaptic muscle fibers were impaled with 3-8 M $\mathrm{M}$ clectrodes. Onc of these was near the presynaptic site $(<100 \mu \mathrm{m}$ away), and one was distant from the presynaptic site $(>2$ mm away; Fig. 1).

When baseline recordings had been obtained, pressure was applied to the presynaptic electrode for injection of the inhibitors into the axon terminals. This was accomplished using a Picospritzer II injection system. Pressure applied to the injection electrode ranged from 5 to 35 pounds per square inch, and in each case was adjusted carefully while the stability of the recordings and the resistance of the presynaptic electrode were monitored. This resistance decreased upon application of pressure, and pressure was adjusted until no further change in electrode resistance was seen. The quality of the electrode and the nature of the material being injected were the most important factors determining the optimal applied pressure. Pressure was applied for $1 \mathrm{hr}$ for PKI and 15 min for SQ22,536; these times were found to be sufficient to ensure adequate entry of the inhibitors, while still retaining localization. RA233 required fairly long injection periods $(1 \mathrm{hr})$ for a molecule of its size $\left(M_{\mathrm{r}} 421.5\right)$. Injection of this material was worked out in experiments with 5-HT; the injection times selected were sufficient to eliminate completely 5-HT facilitation (Dixon, 1988; Dixon and Atwood, 1989). Unfortunately, RA233 could not be localized by fluorescent tagging, so its distribution within the axon and its effective intracellular concentration were not determined.

After injection of any of the above materials, $20-\mathrm{Hz}$ stimulation was administered for $10 \mathrm{~min}$ to induce LTF, and measurements of EPSPs were made as described above (see also Wojtowicz and Atwood, 1986).

PKI was tagged with rhodamine 123 by mixing equal molar concentrations of PKI and rhodamine 123 in the electrode filling solution (without TAPS) at $\mathrm{pH}>10$. After $5 \mathrm{~min}$ the $\mathrm{pH}$ was returned to 7.4 with $\mathrm{HCl}$, and the TAPS buffer was added. Volume was adjusted to give a final PKI concentration of $5 \mathrm{mg} / \mathrm{ml}$.

Following injection and electrical measurements, the preparation was visualized in a fluorescence microscope (excitation filter, $410-485 \mathrm{~nm}$; barrier filter passing $>515 \mathrm{~nm}$ ) and photographed. Figure 2 provides a representative result demonstrating localization of PKI after the experiment.

Fluorescent tagging of PKI did not affect its potency: Similar results were obtained with tagged and untagged PKI. There was no loss of potency of PKI or SQ22,536 in the injection solution for at least several days.

Toxins acting on $G$ proteins. Cholera toxin, "A" subunit (Sigma), which is known to activate $G_{s}$ protein (Gill and Meren, 1978), was dissolved in crayfish solution at a concentration of $100 \mu \mathrm{g} / \mathrm{ml}$. Pertussis toxin (Sigma), known to block $G_{i}$ protein (Katada and Ui, 1982), was dissolved in crayfish solution at a concentration of $2 \mathrm{mg} / \mathrm{ml}$. Both toxins were tested by Dr. P. Pennefather (Faculty of Pharmacy, University of Toronto) on cultured mammalian neurons and were found to be active.
Among other actions, they affected long-term potentiation in the rat hippocampus. They were made up just prior to use in the crayfish preparation.

\section{Results}

Stimulation of the excitor axon at $20 \mathrm{~Hz}$ for $10 \mathrm{~min}$ results in a facilitation of EPSP amplitude, which can be divided into 2 phases (see Figs. 3, 5, 6). The first, or tetanic, phase occurs during and shortly after the 20-Hz stimulus train. EPSP amplitude may increase by as much as $2000 \%$. Thereafter, EPSP amplitude declines rapidly initially, but settles to a level approximately twice the amplitude of the pretetanic EPSP and persists at this level for several hours to $1 \mathrm{~d}$ (long-lasting phase).

\section{Enhancers of CAMP: IBMX and forskolin}

A 5-min application of $1 \mu \mathrm{M}$ forskolin (Seamon and Daly, 1983) rapidly facilitates EPSP amplitude by about $80-100 \%$. Facilitation is maximal by $15 \mathrm{~min}$ after application of forskolin. It then decays over a period of 1-2 hr (Fig. 3). Similar results were seen in 10 experiments.

A 30-min incubation of the preparation in $100 \mu \mathrm{M}$ IBMX (Beavo et al., 1970) produces EPSP facilitation of approximately 200-250\%, which recovers over the course of about $2 \mathrm{hr}$ (Fig. 3). Similar results were obtained in 7 experiments.

Differential incubation times for forskolin and IBMX were selected after preliminary trials showed that these exposure times were necessary to obtain EPSP enhancement. Differences in membrane permeability and other factors may be involved.

Facilitation of EPSPs produced by the above manipulations is similar in amplitude to the long-lasting phase of LTF. However, recovery is evident for IBMX- and forskolin-induced facilitations, but not for LTF.

Forskolin and IBMX have little or no effect on the presynaptic action potential (Fig. 4A). Occasionally, a small depolarization of the axonal membrane potential (about $1 \mathrm{mV}$ ) is evident in conjunction with forskolin application ( 3 of 10 experiments), but not in the example provided. In contrast, during the tetanic phase of LTF, the action potential gets smaller and the membrane hyperpolarizes (Fig. $4 B$ ). These changes are linked to accumulation of sodium (Wojtowicz and Atwood, 1985). The changes are rapidly reversed after stimulation, and no electrical changes remain to account for the long-lasting phase of LTF. 


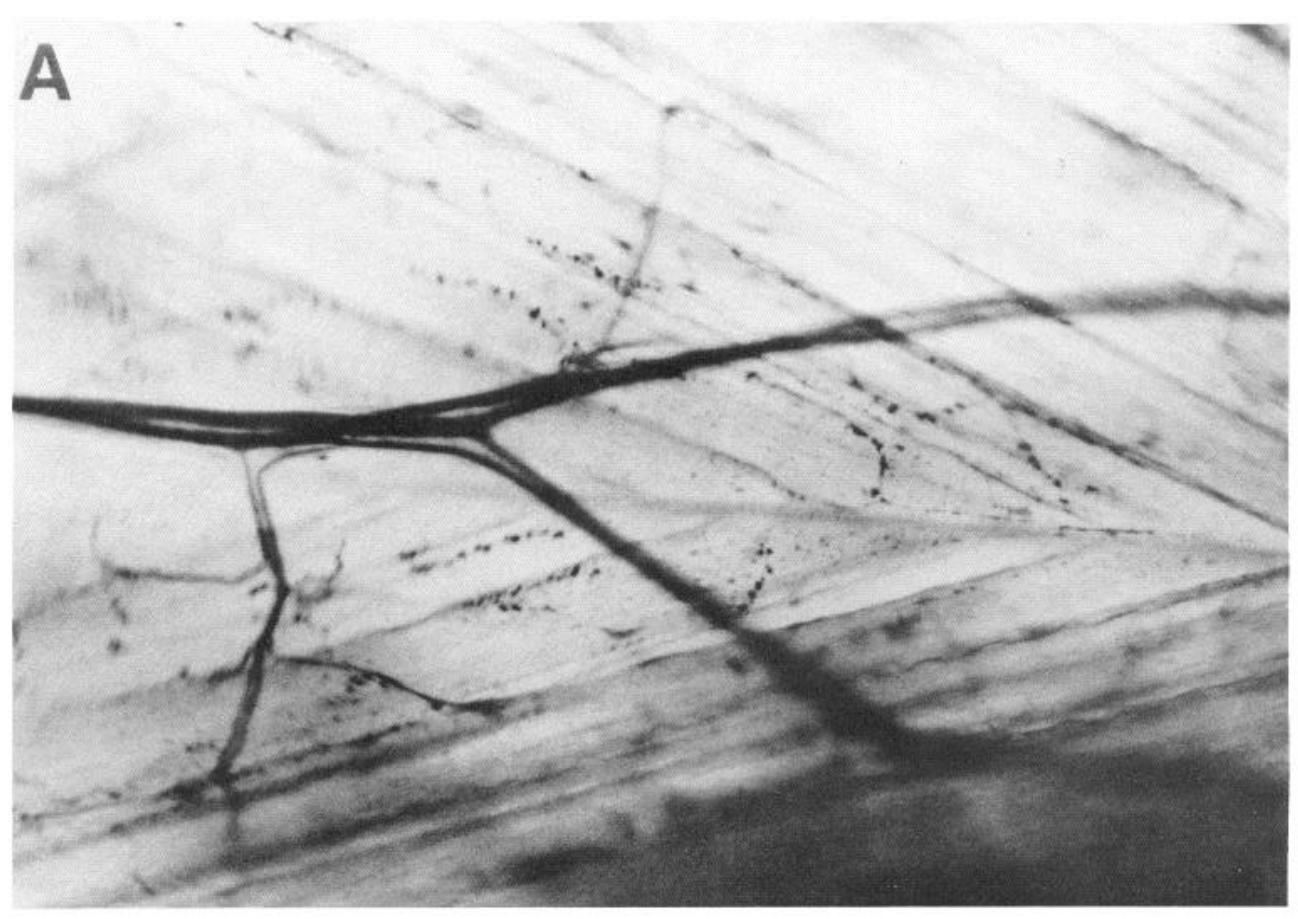

Figure 2. Localization of injected protein kinase inhibitor (PKI). $A$, Typical opener muscle preparation stained with methylene blue to highlight neural tissue is compared with $B$, a preparation in which the excitor axon was injected with rhodamine-tagged PKI (bright structure). The site of injection is indicated by an arrow. The spread of the dye shows the limited distribution of PKI within the axon after injection. The physiological effects of PKI correlate with the limited distribution noted in this figure. Scale: $1 \mathrm{~cm}=75 \mu \mathrm{m}$.

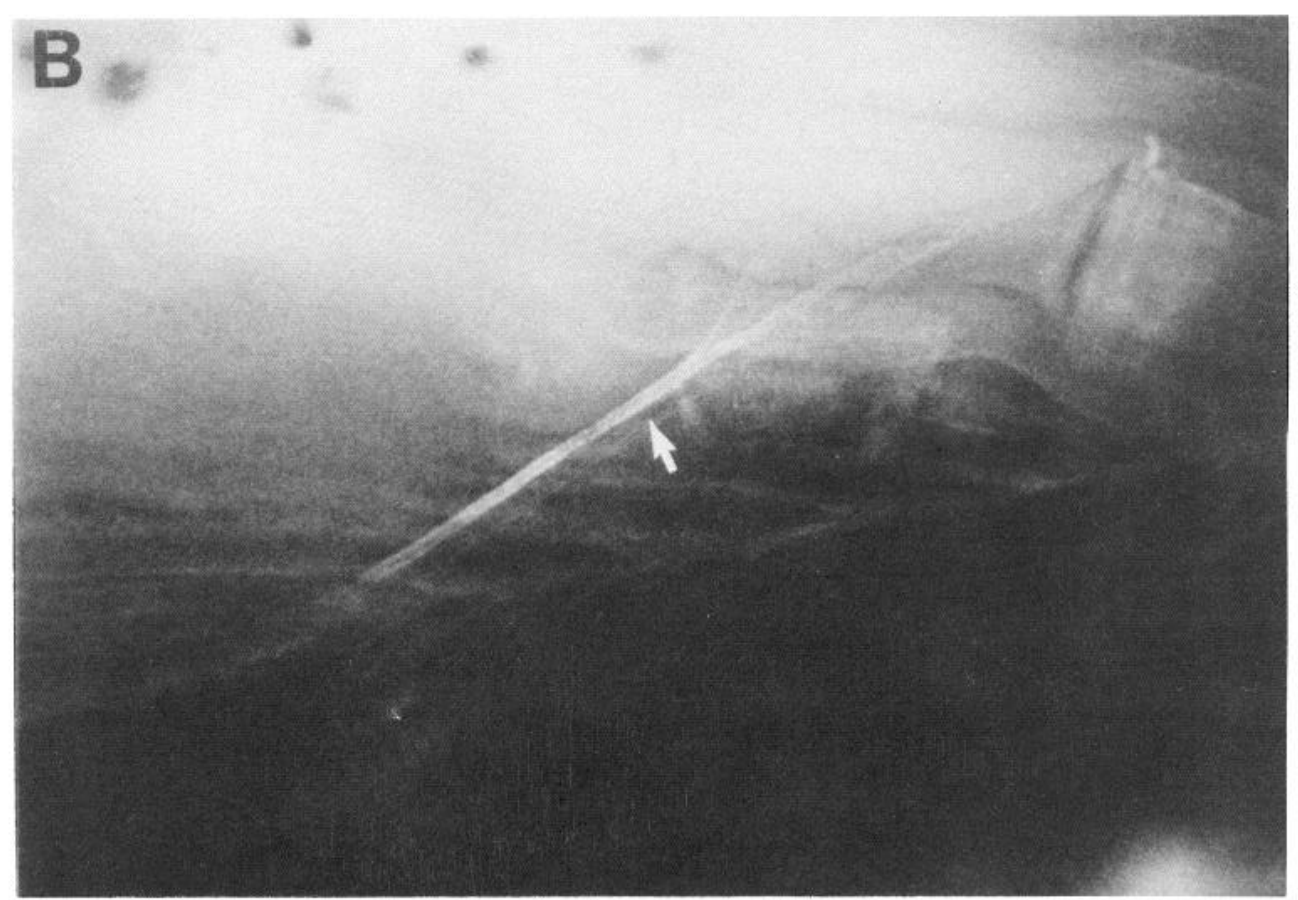

\section{The Walsh Inhibitor (PKI)}

Localized injection of rhodamine-tagged PKI (Walsh et al., 1971; Demaille et al., 1977) blocks the long-lasting phase of LTF at synapses near the site of PKI injection; there is no discernible effect on the tetanic phase. Both phases of LTF occur at synapses of the same axon distant from the injection site (Fig. 5). Localization of the inhibitor was confirmed after the experiments by examining the preparation under a fluorescence microscope and noting the distribution of yellow fluorescence (Fig. 2).

As in the case of forskolin and IBMX, no presynaptic electrical changes could be demonstrated for PKI.
Inhibitor of adenylate cyclase: $S Q 22,536$

Injection of SQ22,536 (adenylate cyclase inhibitor: Harris et al., 1979) prior to $20-\mathrm{Hz}$ stimulation leads to the same result as for the Walsh inhibitor. The long-lasting phase of LTF is inhibited, while the tetanic phase appears normal (Fig. 6). In addition, the effect of SQ22,536 was found to be localized, as in the case of the Walsh inhibitor.

\section{Inhibitor of phospholipase C: RA233}

Injection, prior to $20-\mathrm{Hz}$ stimulation, of RA233 (phospholipase C inhibitor: Hassanein et al., 1970), which blocks initiation of 


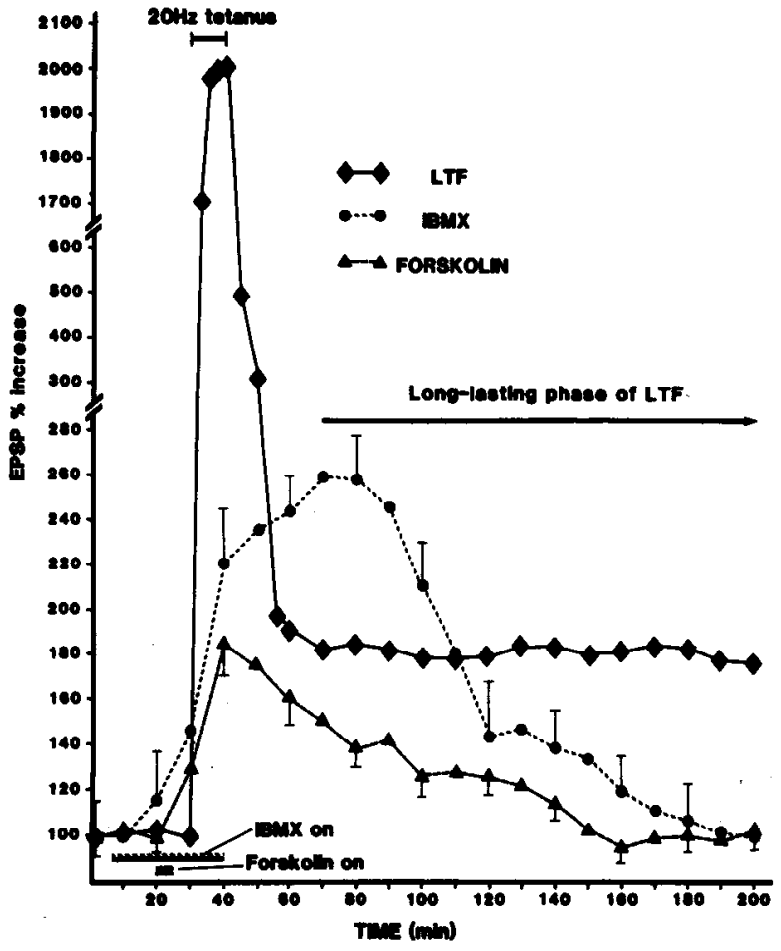

Figure 3. Effects of forskolin (adenylate cyclase activator; 10 experiments averaged) and IBMX (phosphodiesterase inhibitor; 7 experiments averaged). A low-level, long-lasting EPSP facilitation, similar in amplitude to the long-lasting phase of LTF, develops with both agents. A standard graph of LTF is included for comparison. Note that the longlasting phase of LTF is more persistent than facilitation induced by agents that act to elevate cAMP. Error bars indicate standard deviations for combined data for IBMX and forskolin.

the phosphatidylinositol second-messenger system, has no effect on either phase of LTF (Fig. 6).

\section{Toxins acting on $G$ proteins}

Cholera toxin is known to irreversibly activate $\mathrm{G}_{\mathrm{s}}$ (Gill and Meren, 1978), which promotes cAMP production. Incubation

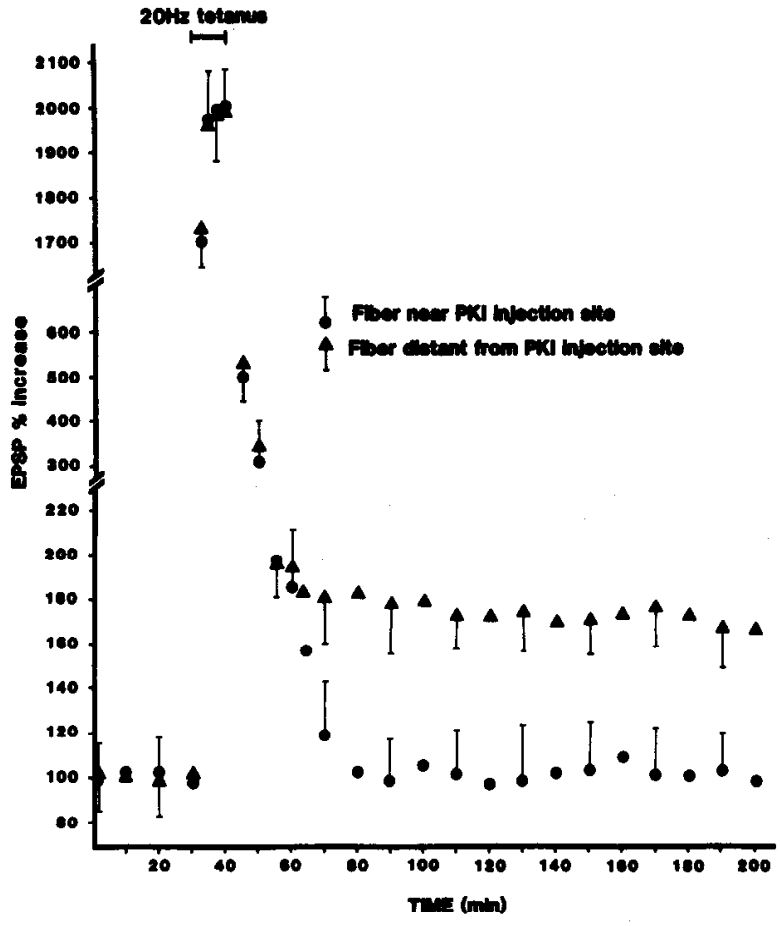

Figure 5. Effects of localized injection of PKI on LTF. Recordings were made from pairs of postsynaptic fibers: one near to and one distant from the presynaptic injection site. All phases of LTF are present at the postsynaptic sites distant from PKI injection ( $\boldsymbol{\Delta}$ ). At the postsynaptic sites close to PKI injection (O), the long-lasting phase of LTF does not develop. Data points are mean EPSP amplitude combined from 6 experiments. The bars indicate the standard deviation for the combined data.

of the opener muscle in cholera toxin (A subunit) for $1 \mathrm{hr}$ results in a doubling of EPSP amplitude, which persists for several hours. Preparations were monitored for $3 \mathrm{hr}$ after the toxin had been washed out, at which time there was no sign of recovery from the toxin's effect on EPSP amplitude. Longer incubations do not enhance the toxin's effect. There are no changes in the
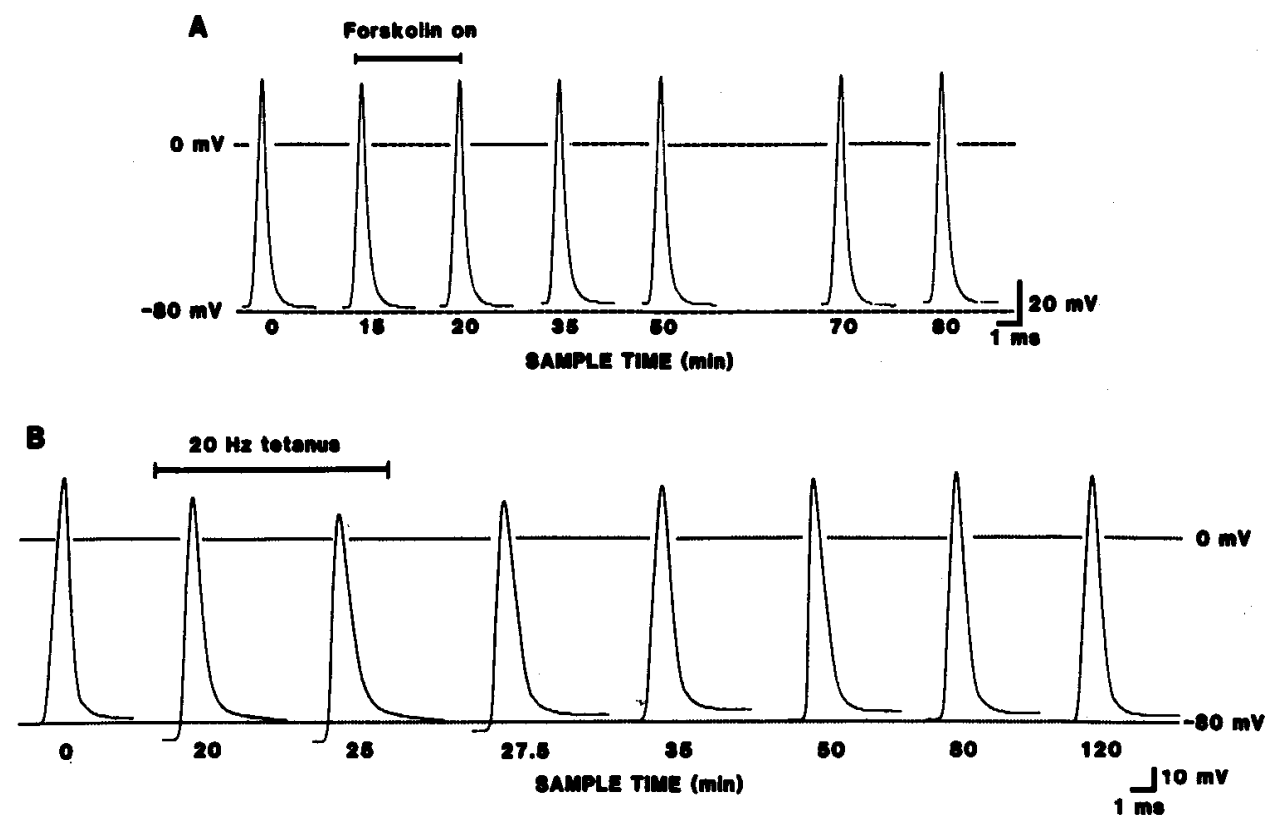

Figure 4. Presynaptic action potentials and axonal membrane potential recorded during application of forskolin $(A)$ and during LTF $(B)$. Forskolin has little, if any, effect on action potential or membrane potential $(A)$. During tetanic stimulation, the action potential decreases in amplitude and the membrane hyperpolarizes, but the changes are rapidly reversed after stimulation. 


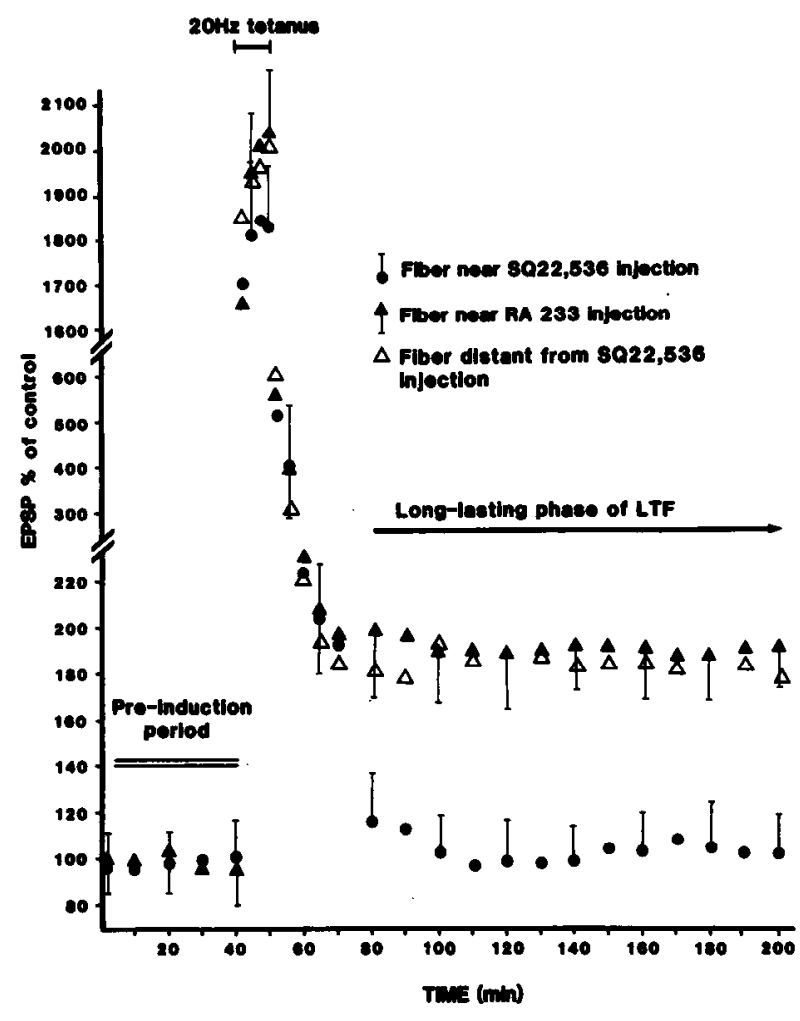

Figure 6. Comparison of the effects of SQ22,536 (adenylate cyclase inhibitor) and RA233 (phospholipase C inhibitor) on LTF. Inhibition of adenylate cyclase prior to $20-\mathrm{Hz}$ stimulation blocks development of the long-lasting phase of LTF (๑). Data points (without error bars) for fibers distant from the site of injection in the same preparations are indicated by open triangles $(\Delta)$. Inhibition of phospholipase $C$ prior to $20-\mathrm{Hz}$ stimulation does not alter any aspect of LTF $(\Delta)$. Data points for each drug treatment are mean EPSP amplitude from 8 SQ22,536 experiments and 5 RA233 experiments. The bars are standard deviations for the combined data for each type of experiment. (Error bars for data points for "distant" fibers from the SQ22,536 experiments are similar to those for the RA233 experiments and are omitted for clarity.)

presynaptic membrane potential or action potential as a result of cholera toxin.

When $20-\mathrm{Hz}$ stimulation is applied to preparations presoaked in cholera toxin for $1 \mathrm{hr}$, LTF still occurs (Fig. 7). However, the percentage increase in EPSP amplitude, especially during the tetanus, is somewhat reduced. This can be attributed to the fact that EPSP amplitude is measured relative to prestimulation as control, and the control baseline is already elevated by treatment with cholera toxin.

Pertussis toxin requires a long incubation period to effectively block the $G_{i}$ protein (Gilman, 1984). Preparations were dissected in the afternoon and left overnight (14-16 hr) in the refrigerator $\left(4^{\circ} \mathrm{C}\right)$ in saline containing $2 \mathrm{mg} / \mathrm{ml}$ pertussis toxin. There were no indications that the overnight soaking adversely affects the preparations. Resting membrane potential, action potential size, EPSP size, and membrane input resistance for both axon and muscle fibers were not significantly different between preparations that had been soaked overnight and those freshly dissected.

Overnight pertussis toxin treatment does not alter any aspect of LTF (Fig. 7). Both pre- and postsynaptic events are normal.

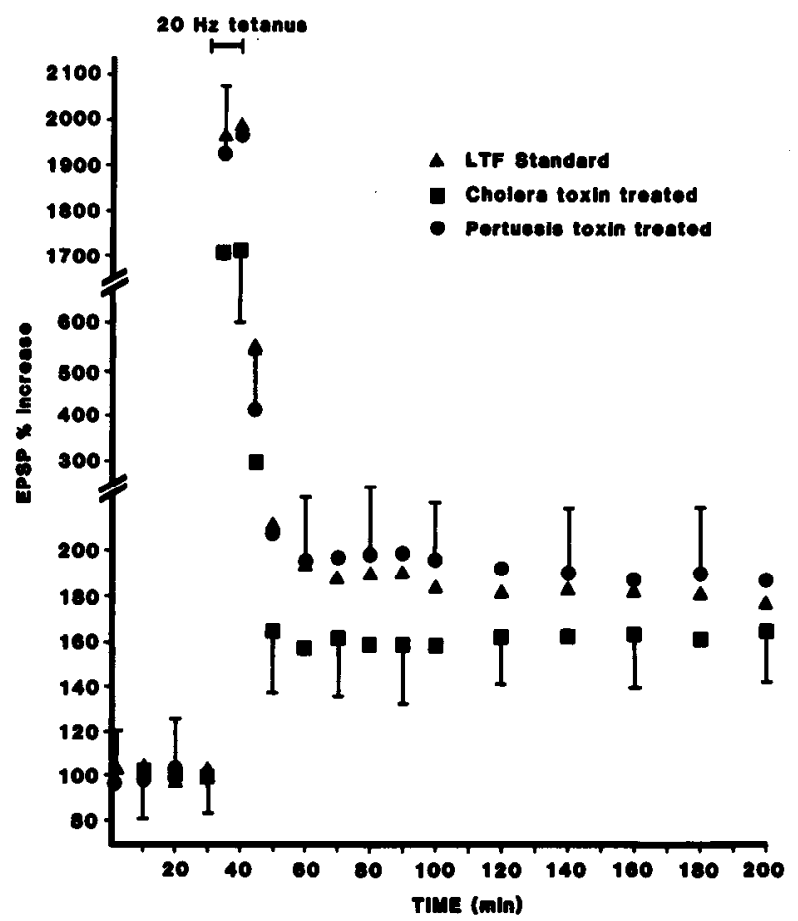

Figure 7. Effects of cholera toxin and pertussis toxin on LTF. Overnight soaking in pertussis toxin (O) has no effect on baseline EPSP amplitude and does not alter LTF. Soaking in cholera toxin $(\square$ for 1 $\mathrm{hr}$ increases EPSP amplitude by $100 \%$ but does not block induction of LTF. The amplitude of LTF is reduced, because the initial (pretetanus) EPSP amplitude has already been increased by cholera toxin. Bars are standard deviation for the combined data in each of 4 experiments for each toxin.

\section{Discussion}

Previous studies of the mechanisms of LTF have investigated the roles played by various ions in establishing the two phases. The consensus is that both sodium and calcium are important during and shortly after the tetanic phase (Atwood and Wojtowicz, 1986). However, the long-lasting phase of facilitation develops despite the absence of sodium and calcium entry during the tetanus (Wojtowicz and Atwood, 1988; Wojtowicz et al., 1988). This suggested to us that a mechanism independent of ion flux must be responsible for the long-lasting phase of LTF.

The present experiments provide two lines of evidence for involvement of the adenylate cyclase pathway in the long-lasting phase of LTF. First, treatments known to enhance cAMP levels produce facilitation similar in amplitude, though shorter in duration. A second, more convincing line of evidence comes from the actions of inhibitors of adenylate cyclase and A-kinase; these compounds block selectively the long-lasting phase of LTF.

The activation of cAMP-dependent protein kinase, through a variety of mechanisms, had been shown previously to produce a low-level facilitation of EPSPs (Enyeart, 1981; Kravitz et al., 1981). Our results confirm these observations. Activation of adenylate cyclase by forskolin or inhibition of cAMP breakdown by IBMX produces a low-level facilitation of EPSPs. The amplitude of such facilitation is similar to that seen during the long-lasting phase of LTF; however, facilitation produced by the pharmacological agents recovers over the course of $1-2 \mathrm{hr}$.

The question of the specificity of forskolin as an agent acting through stimulation of cAMP production has been raised in 
several recent studies that demonstrate reduction of $\mathrm{K}$ currents (specifically $I_{A}$ ) by forskolin in a cAMP-independent manner (Coombs and Thompson, 1987; Baxter and Byrne, 1988; HarrisWarrick, 1988). Our recordings of nerve terminal action potentials showed no evidence of spike broadening, which can readily be produced in this preparation by blocking $\mathrm{I}_{\mathrm{A}}$ with 4-aminopyridine and other agents (e.g., Wojtowicz and Atwood, 1984). Thus, there was no evidence in the present study for a CAMP. independent action of forskolin. However, more direct evidence of changes in cAMP concentration in the axon would be required to decide this point conclusively.

Injection of PKI into a local presynaptic field prior to tetanic stimulation blocks the long-lasting phase of LTF, while the tetanic phase is unaffected. Simultaneous recordings from postsynaptic sites separated by several millimeters of axon show differential facilitation. This ensures that the stimulation was sufficient to establish LTF, and that the absence of LTF at the site close to PKI injection is not an artifact or general failure of expression of LTF in the preparation.

Presynaptic injection of an inhibitor of adenylate cyclase, $\mathrm{SQ} 22,536$, produces the same effect as PKI. Injection has no apparent effect on the tetanic phase of LTF, but blocks expression of the long-lasting phase.

Taken together, the results show that initiation of the longlasting phase of LTF requires CAMP and depends on functional A-kinase. Whether maintenance of the effect also depends on cAMP-mediated reactions remains uncertain. Preliminary experiments aimed at inhibiting the A-kinase after induction of LTF failed for technical reasons. Although no effect on the tetanic phase of LTF could be detected in our measurements, it is possible that the influence of CAMP-mediated reactions is present during this phase. If so, its contribution is buried in the ion-mediated effects, which are known to account for the increased EPSP amplitudes seen during tetanic stimulation (e.g., Wojtowicz and Atwood, 1988).

It is not clear why LTF should last longer than facilitation produced by elevation of CAMP. Enduring EPSP facilitation could result from self-promotion or autophosphorylation intrinsic to the A-kinase (Krebs and Beavo, 1979; Lisman, 1985). The difference in the duration of facilitation produced by IBMX, forskolin, and LTF may lie in the intensity of adenylate cyclase system stimulation produced by each treatment, though there is no way to judge this at present in the absence of measurements of intraaxonal cAMP or other relevant compounds. Another possibility is that the large membrane depolarizations provided by the action potentials during stimulation may lead to persistent alteration of membrane-bound components associated with the adenylate cyclase pathway.

The latter speculation is enhanced by observations suggesting that membrane depolarization, rather than ion fluxes, initiates the long-lasting phase of LTF (Wojtowicz and Atwood, 1988; Wojtowicz et al., 1988). An effect of depolarization on adenylate cyclase activity and A-kinase-dependent protein phosphorylations has been reported in several instances (Browning et al., 1979; Nestler and Greengard, 1980). The link between depolarization and adenylate cyclase activation in the crayfish nerve terminal remains to be determined. However, it is worth noting that work on mammalian brain slices has suggested that electrical stimulation and depolarizing agents can lead to buildup of cAMP, and that $\mathrm{Ca}^{2+}$ is not required for this (Zanella and Rall, 1973; review, Gusovsky and Daly, 1988).

The results obtained with cholera toxin suggest that it was effective in causing a long-lasting enhancement of EPSP amplitude in this preparation and that proportionately less increase in EPSP amplitude could then be induced by $20-\mathrm{Hz}$ stimulation. These results are in accord with a role for the adenylate cyclase system in LTF. The results for pertussis toxin are inconclusive, as no effect was seen and at present there is not a direct measure of possible changes in cAMP level in the axon as a result of treatment.

The present experiments also demonstrate different mechanisms for LTF and for serotonin facilitation. The latter, which is receptor-dependent and less persistent than LTF, involves the phosphatidylinositol second-messenger system (Dixon and Atwood, 1987, 1989; Dixon, 1988). Participation of this system in LTF seems unlikely at present.

\section{References}

Atwood, H. L., and J. M. Wojtowicz (1986) Short-term and long-term plasticity and physiological differentiation of crustacean motor synapses. Int. Rev. Neurobiol. 28: 275-362.

Baxter, D. A., and J. H. Byrne (1988) Reduction of voltage-activated $\mathrm{K}^{+}$currents by forskolin is not mediated by cAMP in pleural sensory neurons of Aplysia. Soc. Neurosci. Abstr. 14: 153.

Beavo, J., N. Rogers, O. Crofford, J. Hardman, E. Sutherland, and E. Newman (1970) Effects of xanthine derivatives on lipolysis and on adenosine 3'5-monophosphate phosphodiesterase activity. Mol. Pharmacol. 6: 597-603.

Browning, M., T. Dunwiddie, W. Bennett, W. Gispen, and G. Lynch (1979) Synaptic phosphoproteins: Specific changes after repetitive stimulation of the hippocampal slice. Science 203: 60-62.

Coombs, J., and S. Thompson (1987) Forskolin's effect on transient $\mathrm{K}$ current in nudibranch neurons is not reproduced by cAMP. J. Neurosci. 7: 443-452.

Demaille, J. G., K. A. Peters, and E. H. Fischer (1977) Isolation and properties of the rabbit skeletal muscle protein inhibitor of adenosine 3',5'-monophosphate dependent protein kinases. Biochemistry 16: 3080-3086.

Dixon, D. (1988) Second messengers in serotonin facilitation and longterm facilitation. Ph.D. thesis, University of Toronto.

Dixon, D., and H. L. Atwood (1987) A role for both cAMP and phosphatidyl inositol in serotonin facilitation in crayfish. Soc. Neurosci. Abstr. 13: 596.

Dixon, D., and H. L. Atwood (1988) Adenylate cyclase activity is required for long-term facilitation. Soc. Neurosci. Abstr. 14: 838.

Dixon, D., and H. L. Atwood (1989) Phosphatidylinositol system's role in serotonin-induced facilitation at the crayfish neuromuscular junction. J. Neurophysiol. 62: 239-246.

Enyeart, J. (1981) Cyclic AMP, 5-HT, and the modulation of transmitter release at the crayfish neuromuscular junction. J. Neurobiol. 12: 505-513.

Gill, D., and R. Meren (1978) ADP-ribosylation of membrane proteins catalyzed by cholera toxin: Basis of the activation of adenylate cyclase. Proc. Natl. Acad. Sci. USA 75: 3050-3054.

Gilman, A. (1984) $\mathrm{G}$ proteins and dual control of adenylate cyclase. Cell 36: 577-579.

Gusovsky, F., and J. W. Daly (1988) Formation of second messengers in response to activation of ion channels in excitable cells. Cell. Mol. Neurobiol. 8: 157-169.

Harris, D. N., M. M. Asaad, M. B. Phillips, H. J. Goldenberg, and M. J. Antonaccio (1979) Inhibition of adenylate cyclase in human blood platelets by 9 -substituted adenine derivatives. J. Cyclic Nucleotide Res. 5: 125-134.

Harris-Warrick, R. M. (1988) Forskolin reduces the potassium current $I_{A}$ by a cAMP-independent action in lobster neurons. Soc. Neurosci. Abstr. 14: 153.

Hassanein, A., A. G. Turpie, G. P. McNicol, and A. S. Douglas (1970) Effect of RA233 on platelet function in vitro. Br. Med. J. 2: 83-86.

Katada, T., and M. Ui (1982) ADP-ribosylation of the specific membrane protein of $\mathrm{C} 6$ cells by islet-activating protein associated with modification of adenylate cyclase activity. J. Biol. Chem. 257: 7210 7216.

Kravitz, E. A., S. Glusman, M. S. Livingstone, and R. M. Harris-Warrick (1981) Serotonin and octapamine in the lobster nervous system: 
Mechanism of action at neuromuscular junctions and preliminary behavioral studies. In: Serotonin, Neurotransmission, and Behavior, B. Jacobs and A. Gelperin eds., pp. 189-210, MIT Press, Cambridge, MA.

Krebs, E. G., and J. A. Beavo (1979) Phosphorylation-dephosphorylation of enzymes. Annu. Rev. Biochem. 48: 923-959.

Lisman, J. E. (1985) A mechanism for memory storage insensitive to molecular turnover: A bistable autophosphorylating kinase. Proc. Natl. Acad. Sci. USA 82: 3055-3057.

Lnenicka, G. A., and H. L. Atwood (1985) Long-term facilitation and long-term adaptation at synapses of a crayfish phasic motoneuron. $\mathrm{J}$. Neurobiol. 16: 97-110.

Montarolo, P. G., P. Goelet, V. F. Castellucci, J. Morgan, E. R. Kandel, and S. Schacher (1986) A critical period for macromolecular synthesis in long-term heterosynaptic facilitation in Aplysia. Science 234 . 1249-1254.

Nestler, E. J., and P. Greengard (1980) Dopamine and depolarizing agents regulate the state of phosphorylation of protein I in the mammalian superior cervical symphathetic ganglion. Proc. Natl. Acad. Sci. USA 77: 7479-7483.

Ross, E. M., and A. G. Gilman (1980) Biochemical properties of hormone-sensitive adenylate cyclase. Annu. Rev. Biochem. 49: 533564.

Schacher, S., V. F. Castellucci, and E. R. Kandel (1988) cAMP evokes long-term facilitation in Aplysia sensory neurons that requires new protein synthesis. Science 240: 1667-1669.

Schwartz, J. H., and S. M. Greenberg (1987) Molecular mechanisms for memory: Second-messenger induced modifications of protein kinases in nerve cells. Annu. Rev. Neurosci. 10: 459-476.

Seamon, K., and J. Daly (1983) Forskolin, cyclic AMP and cellular physiology. Trends Pharmacol. Sci. 4: 120-123.

Sherman, R. G., and H. L. Atwood (1971) Synaptic facilitation: Longterm neuromuscular facilitation in crustaceans. Science 171: 12481250.
Teyler, T. J., and P. DiScenna (1987) Long-term potentiation. Annu. Rev. Neurosci. 10: 131-161.

Van Harreveld, A. (1936) A physiological solution for freshwater crustaceans. Proc. Soc. Exp. Biol. Med. 34: 428-432.

Walsh, D., C. Ashby, C. Gonzalea, D. Calkins, H. Fischer, and E. Krebs (1971) Purification and characterization of a protein inhibitor of adenosine $3^{\prime}, 5^{\prime}$-monophosphate-dependent protein kinases. J. Biol. Chem. 246: $1977-1985$.

Wojtowicz, J. M., and H. L. Atwood (1984) Presynaptic membrane potential and transmitter release at the crayfish neuromuscular junction. J. Neurophysiol. 52: 99-113.

Wojtowicz, J. M., and H. L. Atwood (1985) Correlation of presynaptic and postsynaptic events during establishment of long term facilitation at the crayfish neuromuscular junction. J. Neurophysiol. 54: 220230.

Wojtowicz, J. M., and H. L. Atwood (1986) Long-term facilitation alters transmitter releasing properties at the crayfish neuromuscular junction. J. Neurophysiol. 55: 484-498.

Wojtowicz, J. M., and H. L. Atwood (1988) Presynaptic long-term facilitation at the crayfish neuromuscular junction: voltage-dependent and ion-dependent phases. J. Neurosci. 8: 4667-4674.

Wojtowicz, J. M., I. Parnas, H. Parnas, and H. L. Atwood (1988) Longterm facilitation of synaptic transmission demonstrated with macropatch recording at the crayfish neuromuscular junction. Neurosci. Lett. 90: 152-158.

Zenella, J., and T. W. Rall (1973) Evaluation of electrical pulses and elevated levels of potassium ions as stimulants of adenosine $3^{\prime}, 5^{\prime}$ monophosphate (cyclic AMP) accumulations in guinea pig brain. J. Pharmacol. Exp. Ther. 186: 241-251. 